\title{
Self-Construal and Concerns Elicited by Imagined and Real Health Problems ${ }^{1}$
}

\author{
Ayse K. UsKuL ${ }^{2}$ \\ University of Michigan \\ Ann Arbor, MI
}

\author{
Michaela Hynie \\ York University \\ Toronto, Ontario, Canada
}

\begin{abstract}
In 2 studies, we examined the relationship between self-construal and illness-related concerns. In Study 1, participants imagined themselves experiencing a health problem described in a scenario and answered closed-ended questions about the concerns that this situation would likely elicit. The experience of social illness concerns was predicted by collective self-construal, and the experience of personal illness concerns tended to be predicted by endorsement of individual self-construal. In Study 2, participants recalled a past health problem and related consequences, which were content-coded. Collective self-construal predicted the extent to which people mentioned issues related to others in their free-recall illness descriptions and the number of other-related consequences that were generated when specifically asked about them.
\end{abstract}

Sarah falls ill. She is told that she must stay in bed for a while to get better. She starts worrying, because staying in bed might mean not being able to finish her ongoing project at work, thereby putting her at a disadvantage for the promotion for which she has been hoping. She also feels that lying in bed threatens her independence, as she dislikes being dependent on other people for her needs. Julie, who is also ill, is told that she needs to rest in bed in order to recover more quickly. She is worried because her mother must take time off work to care for her. She feels that she is a burden to her family. In addition, this situation prevents her from being able to take care of her younger sister.

When people experience a health problem, they tend to feel concerned about its consequences. Their concerns may be related to how they will catch

${ }^{1}$ The authors thank Lynda Cheng, Adele Ambrose, Uzma Jeelani, Andy Ng, Mandeep Singh, Marina Veprinska, and Syeda Abedi for their assistance in conducting the studies and coding the open-ended data; and Sunyoung Jung for her help in preparing the manuscript. Finally, thanks go to Lilach Sagiv for her valuable comments on an earlier draft of the manuscript. This research was supported by a doctoral fellowship from the Ontario Women's Health Council, Canada to Ayse Uskul. Uskul was funded by a postdoctoral fellowship of the Social Science and Humanities Research Council of Canada while working on the manuscript.

${ }^{2}$ Ayse K. Uskul is now at the University of Essex. Correspondence concerning this article should be addressed to Ayse K. Uskul, University of Essex, Department of Psychology, Wivenhoe Park, Colchester, CO4 3SQ United Kingdom. E-mail: auskul@essex.ac.uk

\section{6}

Journal of Applied Social Psychology, 2007, 37, 9, pp. 2156-2189.

(C) 2007 Copyright the Authors

Journal compilation (C) 2007 Blackwell Publishing, Inc. 
up with work, how they will do on the next exam, how much work their illness will create for others, or whether they will be able to fulfill their social responsibilities. The concerns that emerge in one's mind as a result of being ill can be powerful in directing one's subsequent thoughts and actions, such as health information seeking, adherence to treatment, seeking social support, interpretation of symptoms, or coping with illness. For example, Sarah might decide not to adhere to her physician's recommendation to stay in bed because of her fear of not being able to finish her work project. Similarly, Julie might decide to continue taking care of her sister, even though she has been advised to stay in bed for several weeks. The behavior in which both Sarah and Julie engage might be the same; namely, not adhering to a physician's recommendations, but the underlying reasons might differ. Therefore, different strategies may be needed to convince them to engage in the recommended behavior.

A potential factor that we believe would have an impact on the nature of concerns that one would experience in the face of a health problem is selfconstrual; namely, the extent to which significant others are incorporated in the organization of one's self-concept (Markus \& Kitayama, 1991). Although self-construal has been used frequently to explain various social psychological phenomena (e.g., self and other perception, causal attributions, conflict resolution), it has rarely been examined in the context of how people think of and experience physical health problems. We believe that knowing how illness-related concerns are shaped as a function of one's self-construal will contribute to our understanding of the role that the self plays in illness and provide us with new ideas to be applied in the domain of the psychology of health and illness.

The present studies report on whether there is a link between the nature of illness concerns and self-construal when one imagines oneself suffering from a physical health problem (Study 1) and when one experiences an actual health problem (Study 2). First, we will describe how we conceptualize illness concerns; then we will describe self-construal; and finally, we will explain why we are interested in the link between illness concerns and self-construal.

\section{Illness Concerns}

The terms concerns or worries have generally been conceptualized as subtypes of anxiety, whose presence serves as an indicator of poor mental health (for a review, see Davey \& Tallis, 1994). In a significant departure from this view, Boehnke and his colleagues (Boehnke, Schwartz, Stromberg, \& Sagiv, 1998) distinguished between micro worries, which focus on the self and are related to poor mental health, and macro worries, which focus on 
others and are not related to poor mental health. Concerns have also been conceptualized as an aspect of emotions together with appraisal, action readiness, social sharing, and belief changes (Frijda, 1986; Lazarus, 1991). According to Mesquita (2001), when experiencing interdependent emotions (e.g., shame), one's concerns focus on one's own social worth and the worth of the in-group. When experiencing independent emotions (e.g., anger), however, the focus is on personal issues.

In the current paper, we examine specific concerns evoked in relation to a physical health problem, which can be thought of as people's worries about related potential consequences. This conceptualization is similar to one of the five attributes of illness representation included in Leventhal and colleagues' self-regulation model (Leventhal, Diefenbach, \& Leventhal, 1992; Leventhal, Nerenz, \& Steele, 1984); namely, consequences of illness. The other attributes of the model - namely, identity, timeline, antecedent causes, and potential for cure or control - tend to focus on the physical illness itself, whereas consequences of the illness can be not only physical, but also economic, social, and interpersonal (Bishop, 1987; Meyer, Leventhal, \& Gutmann, 1985). Since consequences of illness can have implications for the individual who experiences the physical health problem as well as other people who are related to this individual, the characteristics of one's self-construal will be more likely to have an impact on the nature of concerns or worries about possible consequences of illness than on other components of illness representation.

\section{Self-Construal}

The self literature typically discusses how individuals construe the self in two distinct ways. One type of construal is described by reference to concepts such as individualisic, independent, autonomous, agentic, and separate; and the other by reference to their bipolar ends, such as collectivistic, interdependent, ensembled, communal, or relational (Bakan, 1966; Gilligan, 1982; Kashima et al., 1995; Markus \& Kitayama, 1991; Triandis, 1989). The first type of self-construal, which is considered to be prevalent among men and in Western cultures, is viewed as autonomous, separate, and unique. For individuals who endorse this type of self-construal, the focus is on the personal. The boundary between cognitive representations of the self and others is clear-cut, and the self is constructed on the basis of abilities, traits, and attributes (Cross \& Madson, 1997; Kanagawa, Cross, \& Markus, 2001). These individuals are also likely to strive for dominance, mastery, and power, which enhance and protect the differentiation of the individual (Wiggins, 1991).

The second type of self-construal, which is considered to be prevalent among women and in most non-Western, collectivistic cultures, is viewed as 
embedded within the social context and defined by social relations, membership in groups, and social positions (Markus \& Kitayama, 1991; Shweder \& Bourne, 1982; Triandis, 1989). For individuals who endorse this type of self-construal, the focus is on the self in relation to others. The boundaries between cognitive representations of the self and important others are flexible or permeable, and the behaviors of such individuals are guided by the perceived thoughts, feelings, and behaviors of important others (Markus \& Kitayama, 1991). These individuals are also likely to strive for intimacy, connectedness, and solidarity with a social entity (Wiggins, 1991) and are highly motivated by the need to care for others (Bakan, 1966; McAdams, 1995).

Following research that distinguished the type of interdependent selfconstrual prevalent in non-Western cultures versus Western cultures (e.g., Kashima et al., 1995), a three-dimensional view of self-construals has been receiving growing attention. This perspective introduces a distinction between individual, relational, and collective aspects of the self (Cross \& Madson, 1997; Kashima et al., 1995). Individual self involves the concept of the self as autonomous and unique, having a clear boundary from others (Bakan, 1966; Geertz, 1975; Loevinger, 1976). It is thought to be associated with personal agency, independence from others and the social context, and a belief that the self is not similar to others (Kashima et al., 1995; Triandis, 1995; Triandis, McCusker, \& Hui, 1990). Relational self reflects selfdefinitions derived from ties with specific others, the quality of these relationships, one's interpersonal roles, and characteristics shared with significant others (Aron, Aron, \& Smollan, 1992; Cross \& Madson, 1997; Gilligan, 1982). The relational self is thought to be associated with interpersonal relatedness, intimacy, and interdependence (Baumeister \& Leary, 1995). Collective self refers to self-definitions derived from one's memberships in in-groups or social categories and is thought to be associated with an emphasis on group affiliation, in-group norms, and roles and status defined by collectives (Tajfel \& Turner, 1979; Triandis, 1995).

Research has suggested that differences in self-construal types cannot be treated purely as characteristics of certain cultural or gender groups. There is evidence suggesting that within-culture variability may be greater than between-culture variability, and all types of self-construal may be observed in men and women to some degree (e.g., Brockner \& Chen, 1996; Gudykunst et al., 1996; Oyserman, Coon, \& Kemmelmeier, 2002; Uskul, Hynie, \& Lalonde, 2004). In fact, a basic assumption in the self-construal priming literature is that across societies and cultures, individuals are capable of thinking about themselves and the world as both separate and independent and as connected and interdependent, even if they are typically likely to focus on one or the other. Therefore, either independent or interdependent self- 
focus can be brought to mind for anyone, depending on contextual demands (Oyserman \& Lee, in press).

Research has thus suggested that despite the typological approach, which dominates the literature on self-construal, differences in self-construal are a matter of degree, rather than categorical (for a review, see Oyserman et al., 2002). For example, it is not the case that Americans or men hold an independent self, but do not endorse an interdependent self at all. Similarly, Japanese or women do not show the exact opposite of this pattern. It is likely that social structures and situations in a given culture provide sufficient experience of interdependence and independence for individuals to develop cognitive and social tools to deal with both types of situations, even though the cultural context renders the use of an independent or interdependent lens more functional, given the dominant set of demands that culture puts on its members.

From a between-groups perspective, because of cultural differences in predominant experiences, groups can differ on independence or interdependence. From a within-individual perspective, individuals can be high on independence and interdependence, low on both, or one orientation can dominate the other. In the studies reported here, we examined self-construal as an individual-difference variable and conducted our studies in Toronto, Canada, where the composition of the city population is truly heterogeneous in terms of cultural background (Jiménez \& Lunman, 2004).

Why Examine the Link Between Illness Concerns and Self-Construal?

Different types of self-construal have been researched in relation to physical and psychological well-being, with a focus on the distinction between agency, which is associated with autonomy and a focus on oneself, and communion, which is associated with interdependence and a focus on social others and relationships. Extreme agency (i.e., unmitigated agency) is characterized by a primary focus on oneself at the expense of exclusion of others and is shown to relate to emotional inhibition and hostility, which have been associated with interpersonal difficulties and physical health problems (e.g., cardiovascular disease; for a review, see Smith, 1992). Extreme communion (i.e., unmitigated communion) involves focusing on others' problems and needs to the neglect of oneself. This overinvolvement with others can lead to psychological stress (e.g., Helgeson \& Fritz, 1996) and poor health practices, such as failure to adhere to a heart-healthy diet (Fritz, 2000; Helgeson, 1990, 1995). Thus, individualistic elements in the construal of the self may lead to different health-related consequences than will relational or collectivistic elements. 
In the current studies, we examine the psychological influences of facing a health problem in the form of concerns experienced as a function of one's self-construal. Illness often creates barriers to physical performance and turns attention toward social and behavioral capacities that may be lost or threatened by one's physical condition (Lipowski, 1985). Illness can prevent people from fulfilling their self-defining social roles. It can lead them to a belief that they cannot maintain a state of affairs that is important to them, and that they ultimately will be in a position that diverges from their actual as well as their ideal self-definition. Thus, the specific content of individuals' concerns is likely to reflect what is important to them and what is central to their sense of self (Boehnke, Stromberg, Regmi, Richmond, \& Chandra, 1998). In essence, illness can be experienced as a threat to the self, and may evoke significant concerns about the aspects of the self that are most central and least separable. It follows, then, that concerns elicited by an illness threat would be expected to differ according to what is self-defining.

Because of their embeddedness in social relations and group memberships, people with an interdependent self-construal may be more concerned about the social or interpersonal consequences of illness, such as the effects of the illness on others or changes in relationships. In contrast, people with highly independent self-construals, who view themselves as separate from the social context and from their relationships, may be more likely to think about individual consequences of illness and the ways in which illness affects their own behavior, well-being, or self-conceptions. Thus, the main purpose of the present studies is to examine the extent to which the incorporation of significant others in the organization of the self-concept affects a person's illnessrelated concerns.

In examining our research question, we also took into account the nature of the health problem. The characteristics associated with different health problems may serve to focus people's attention more on themselves or on those around them. For this reason, in Study 1, we manipulated the characteristics of the health problem being described. Specifically, we distinguished between health problems with characteristics likely to lead to increased dependency on others and those with characteristics likely to lead to increased interference with one's own life. We expected that health problems of the former type (i.e., dependency on others) may increase concerns about consequences of a health problem for social others. Individuals who define themselves in relation to others may be more likely to experience such concerns if that health problem is likely to lead to relational consequences. Health problems of the latter type (i.e., interference with one's own life), however, may increase concerns about consequences of a health problems for personal matters. Individuals who are self-focused may be more likely to 


\section{2}

experience self-related concerns if their health problems are likely to lead to consequences primarily detrimental for this personal life.

\section{Study 1}

The goal of Study 1 is to examine the relationship between self-construal and the content of concerns that people develop following illness. We asked participants to imagine suffering from a particular health problem by reading a scenario that describes either back pain or dizziness. The scenarios varied according to level of interference and dependence that the health problem causes in the individuals' lives. In our examination of illness concerns and self-construal, we incorporate the tripartite view of self-construal that distinguishes between individual, relational, and collective self-construal (e.g., Brewer \& Gardner, 1996; Kashima et al., 1995). We propose the following hypotheses:

Hypothesis 1. Endorsement of individual self-construal will be associated with illness concerns related to oneself.

Hypothesis 2. Endorsement of relational self-construal will be associated with illness concerns related to one's close others.

Hypothesis 3. Endorsement of collective self-construal will be associated with illness concerns related to the social groups to which one belongs.

Hypothesis 4. Health problems that cause a high level of interference will be associated with greater experience of concerns related to oneself.

Hypothesis 5. Health problems that cause a high level of dependence will be associated with greater experience of concerns related to others.

We also explore the moderating role of independent and interdependent self-construals on the hypothesized effects of high and low levels of interference and dependence on different kinds of concerns.

\section{Method}

\section{Participants}

Participants were 269 undergraduate students (78 men, 190 women; 1 participant failed to identify gender) who were recruited from different 
psychology courses on a voluntary basis and using the university research participant pool. In exchange for their participation, they each received half a credit. One third (31.6\%) of the participants self-identified as European, $20.1 \%$ as Canadian, $17.0 \%$ as East Asian, $7.4 \%$ as South Asian, $14.0 \%$ as either Caribbean or African, and $8.6 \%$ as Middle Eastern. There were 5 participants who failed to identify their ethnicity.

\section{Procedure}

The study was described to participants as a survey of illness-related concerns. Participants read an illness scenario and answered illness-related concerns while imagining themselves in the situation described in the scenario. They then completed a self-construal scale, followed by questions on demographics.

\section{Study Materials}

Illness scenarios. The questionnaire included one of eight different illness scenarios. There were four scenarios that present a back pain example, while the other four present a dizziness example. There were two levels (high vs. low) of interference and two levels (high vs. low) of dependence that were fully crossed across scenarios. The following is an example of the back-pain scenario with low levels of dependence and low levels of interference:

Imagine that you had been suffering from back pain that was caused by a small lump in your spinal cord. Although the lump was successfully removed, the aftereffects of the operation caused some difficulties in walking and moving around, but you are still able to function in your daily life. Your physician has told you that you should avoid carrying heavy things, walking long distances, and especially driving for the rest of your life since these would be potential triggers for a future back problem. However, you don't expect this to be a big problem since you live in a neighborhood with easy access to public transportation.

In the high-interference condition, the first italicized section was replaced with "that interfere with your daily life" to emphasize that the problem was preventing the person from continuing his or her daily life as before. In the high-dependence condition, the second italicized section was replaced with "This will make you very reliant on others because you live in a neighborhood 
with little access to public transportation" to emphasize that the person would be dependent on others for transportation and, consequently, for doing many things in life.

The scenarios were pilot-tested before they were employed in this study. Participants (22 women, 9 men) rated high-interference scenarios as significantly more interfering $(M=5.98)$ with one's life than low-interference scenarios $(M=4.72), F(1,28)=24.13, p<.001$. They also rated highdependency scenarios as leading to significantly more dependency $(M=4.09)$ than low-dependency scenarios $(M=5.86), F(1,28)=48.97, p<.001$.

Illness concerns. A list of 37 illness concern items was developed to tap into personal, interpersonal, and group-related concerns. The items were developed following a thorough examination of existing individualism/ independence and collectivism/interdependence scales, in addition to independent and interdependent self-construal scales (e.g., Hui, 1988; Matsumoto, Weissman, Preston, Brown, \& Kupperbusch, 1997; Singelis, Triandis, Bhawuk, \& Gelfand, 1995; Triandis et al., 1986; Triandis \& Gelfand, 1998). However, in order to prevent replication in the illness concerns scale, the self-construal scales employed in this study were not used in the generation of illness concern items.

Concern items were introduced with the statement "In such a situation I would be concerned about...". There were 15 items designed to assess personal concerns (e.g., "not being able to rely on myself"); 11 items to assess concerns related to close others (e.g., "being a burden on people who are close to me"); and 11 items to assess group-related concerns (e.g., "not being useful in the social group(s) I belong to"). Participants were instructed to respond to the items containing "social groups" by thinking of a social group of which they are a part, such as a religious group, student activity club, or ethnic community. They responded on a 7-point Likert-type scale ranging from 1 (not at all) to 7 (extremely).

Relational-Individual-Collective Self-Aspects Scale (RIC). The RIC combines the measurement of relational, individual, and collective self-construal in three subscales. This scale, developed by Kashima and Hardie (2000), consists of 10 statements, each followed by three options reflecting the three self-aspects. Respondents rate each option in terms of its applicability to the self using a 7-point scale ranging from 1 (not like me, not true of me) to 7 (like me, very true of me). A sample statement is "I think it is important in life to ..." and the three options reflecting the three self-aspects are "have good personal relationships with people who are important to me" (relational; RIC-R), "have personal integrity/be true to myself" (individual; RIC-I), and "work for causes to improve the well-being of my group" (collective; RIC-C). The results yield three subscale scores revealing the relative prominence of each self-aspect. Reliability coefficients were as follows: RIC-R, $\alpha=.69$ 
$(M=5.94, S D=0.59) ;$ RIC-I, $\alpha=.67(M=5.96, S D=0.64) ;$ and RIC-C, $\alpha=.78(M=5.24, S D=0.89)$.

Demographics. Participants answered questions about their age, sex, and ethnicity.

\section{Results}

\section{Illness Concerns Scale}

Principal axis factoring was used to conduct an exploratory factor analysis on the illness concern items. A scree plot, used as the criterion to determine the number of factors to retain, clearly suggested a two-factor solution. The factor analysis was repeated, forcing a two-factor solution and using an oblique rotation. Seven out of 11 interpersonal illness concern items and 10 out of 11 group-related illness concern items loaded on the first factor, with factor loadings ranging between .47 and .85 ; whereas the remaining items had either a poor loading or loaded equally well on both factors. Eleven out of 15 personal illness concern items loaded on the second factor, with factor loadings ranging from .54 to .96 (see Appendix for scale items). The remaining items again either had a poor loading on this factor or loaded equally well on both factors. This two-factor solution accounted for $53.7 \%$ of the total variance, with the first factor accounting for $47.1 \%$ of the total variance and the second factor accounting for an additional $6.6 \%$. The factor analysis was repeated with centralized item scores, and the same pattern of factor structure and correlation between factors was obtained.

Two subscales were formed, following the factor structure, by averaging the items in each factor that clearly loaded on one of the two factors. One subscale was called the personal illness concerns subscale, and the other was called the social illness concerns subscale. The former had 11 items and a reliability of $.93(M=5.32, S D=1.22)$. The latter consisted of 17 items and had a reliability of $.94(M=4.27, S D=1.26)$. These two scales were highly correlated $(r=.74, p<.001$; see Table 1 for all correlations).

\section{Illness Concerns and Self-Construal}

To examine the relation between the criterion variables (personal illness concerns and social illness concerns) and the predictors (RIC self-construals, interference, and dependence), two separate hierarchical regression analyses were conducted. Following Aiken and West (1991), the two categorical predictors (i.e., interference and dependence) were effect-coded, the three con- 
Table 1

Correlations Between Illness-Related Concerns and Self-Construal Scales: Study 1

\begin{tabular}{lcccccc}
\hline Variable & 1 & 2 & 3 & 4 & 5 & 6 \\
\hline 1. Social illness concerns & - & & & & & \\
2. Personal illness concerns & $.74^{* *}$ & - & & & & \\
3. RIC-I (individual) & .06 & $.23^{* *}$ & - & & & \\
4. RIC-R (relational) & $.27^{* *}$ & $.25^{* *}$ & $.44^{* *}$ & - & & \\
5. RIC-C (collective) & $.29^{* *}$ & .09 & $.18^{* *}$ & $.51^{* *}$ & - & \\
6. Interference & .10 & .06 & $.13^{*}$ & .10 & .004 & - \\
7. Dependence & .09 & $.16^{*}$ & .03 & -.02 & -.01 & $-.14^{*}$ \\
\hline
\end{tabular}

Note. $\mathrm{RIC}=$ Relational-Individual-Collective Self-Aspects Scale.

$* p<.05 .{ }^{* *} p<.01$.

tinuous predictors (i.e., relational, individual, and collective self-construal scores) were centered, and the product terms were created for two-way interactions. The main effects were entered in Step 1 of the regression, followed by the interaction terms between self-construal scores and the two categorical variables in Step 2. Data were analyzed with and without sex. Because the results did not change with its inclusion, sex was excluded from the following analyses.

The first hierarchical regression with social illness concerns as the criterion revealed an $R^{2}$ significantly different from 0 at the end of Step 1 only $\left(R_{\text {adj. }}^{2}=.11\right), F(5,263)=7.61, p<.001$. The two significant predictors in Step 1 were the RIC-R and RIC-C (see Table 2 for regression coefficients) such that the endorsement of both the relational and collective self-construal was positively associated with the experience of social illness concerns. The level of dependence depicted in the illness scenario contributed marginally significantly to the prediction of the experience of social illness concerns, such that receiving the illness scenario with high dependence tended to be associated with greater experience of social illness concerns. The addition of the interaction terms in Step 2 did not significantly contribute to explanation of the variance $\left(R^{2} \Delta=.02, p=.63\right)$.

The second hierarchical regression with personal illness concerns as the criterion revealed a significant $R^{2}$ in Step 1 only $\left(R_{\text {adj. }}^{2}=.09\right), F(5,263)=6.38$, $p<.001$. The two significant predictors in Step 1 were RIC-R and the level of dependency depicted in the scenario. RIC-I had a marginally significant contribution (see Table 2 for regression coefficients). These effects show that 
Table 2

Summary of Hierarchical Regression Analysis Examining the Relationship Between Illness Concerns and Self-Construal Scores

\begin{tabular}{llrrc}
\hline Dependent measure & Predictors in Step 1 & \multicolumn{1}{c}{$\beta$} & \multicolumn{1}{c}{$t$} & $p$ \\
\hline Social illness concerns & RIC-I (individual) & -.077 & -1.185 & .237 \\
& RIC-R (relational) & .195 & 2.646 & .009 \\
& RIC-C (collective) & .202 & 3.002 & .003 \\
& Dependence & .112 & 1.927 & .055 \\
& Interference & .103 & 1.743 & .083 \\
& $F(5,263)=7.61, p<.001, R=.36, R_{\text {adj. }}^{2}=.11$ \\
Personal illness concerns & RIC-I & .126 & 1.928 & .055 \\
& RIC-R & .221 & 2.970 & .003 \\
& RIC-C & -.047 & -.693 & .489 \\
& Dependence & .163 & 2.767 & .006 \\
& Interference & .044 & 0.736 & .463 \\
& & $F(5,263)=6.38, p<.001, R=.33, R_{\text {adj. }}^{2}=.10$ \\
\hline
\end{tabular}

Note. Only the first step in both regressions is presented. RIC= RelationalIndividual-Collective Self-Aspects Scale.

the endorsement of relational and individual self-construal and a high level of dependence depicted in the illness scenario were positively associated with the experience of personal illness concerns in a hypothetical illness context. The addition of the interaction terms in Step 2 did not significantly contribute to the explanation of variance $\left(R^{2} \Delta=.03, p=.20\right)$.

\section{Factor Structure of Self-Construal Measures}

Both regressions reported previously reveal relational self-construal as a significant predictor of both personal and social illness concerns. We examined this unexpected finding by factor-analyzing self-construal scale scores to test whether these three scales measured three separate constructs, as intended. A scree plot in a principal axis factor analysis suggested two factors. After an orthogonal rotation, it was found that the collective selfconstrual scale loaded on the first factor with a factor loading of .96, clearly separated from the individual self-construal factor, which loaded on the 
second factor with a factor loading of .94. The relational self-construal scale, however, had very similar loadings on both factors (.61 and .51). This factor structure suggests that relational self, as measured by the RIC-R, relates to both individual and collective aspects of the self.

\section{Discussion}

In Study 1, we examined the relationship between self-construal and type of illness concerns. We asked participants to imagine themselves experiencing a health problem described in a scenario and to answer closed-ended questions about the concerns that this situation would likely elicit.

\section{Illness Concerns and Self-Construal}

Factor analysis of illness concern items suggests a two-factor solution in which most of the interpersonal and group-related concern items loaded on one factor separate from most of the individual concern items, which loaded on a second factor. These two factors were highly correlated, whereby participants who scored high on personal illness concerns also scored high on social illness concerns. This high positive correlation may be explained by a response bias as a result of one-directional phrasing of all items in the scale resulting in lack of items with a reversed meaning. However, the fact that the factor analysis reveals two separate factors suggests that response bias cannot be entirely responsible for the observed correlation. Also, it is reasonable that people who have a tendency to worry about the consequences of healthrelated problems (Lucock \& Morley, 1996) may have concerns in both individual and social domains.

The lack of differentiation between responses to concerns that refer to one's social groups and concerns that refer to one's close relationships might have stemmed from an overlap between participants' close others and social groups. When responding to illness concern items, participants in this study, who were undergraduate students, may have thought of their family members as close others in their life and as their primary social group. For young adults, parents may continue to be primary attachment figures, because they have not yet established very close relationships with others (Youniss \& Smollar, 1985). A second potential explanation could be that the social groups of which young adults are members are also often where they make their friendships.

As hypothesized, the regression analyses reveal that collective selfconstrual significantly predicted the experience of social illness concerns, and 
individual self-construal tended to predict the experience of personal illness concerns. Relational self-construal predicted the experience of both types of illness concerns. This may be a product of the characteristics of the RIC scale, which asks respondents to rate all three aspects of the self in each question, a potential factor that might have led to a lack of differentiation between the relational and the other two types of self-construals. A conceptual explanation for potential overlap between the relational and collective self put forward by Kashima and colleagues (Kashima, Kashima, \& Aldridge, 2001) suggests that these two selves may have conceptual similarities to the extent that a group contains significant interpersonal relationships. The overlap between the relational and individual self may be a result of the fact that defining oneself in relation to others may nourish the individual self because relationships are also known to serve the self (Aron \& McLaughlin-Volpe, 2001).

In sum, the prediction of social and personal illness concerns by collective and individual self-construals, respectively, suggests that although health problems are experienced as physically affecting only one person, some people - namely, those who define themselves in collective terms by their membership in social categories - tend to be concerned more with how their illness might affect close others and their in-groups. Moreover, it is more likely for individuals who define themselves as autonomous and unique and who have a clear boundary from others to experience illness concerns related to personal matters.

\section{Effects of Dependence Caused by Illness}

The level of dependence manipulated in the illness scenarios tended to have an effect on the reporting of both types of illness concerns. In addition, this effect was found to be independent of the type of self-construal that one endorses. A potential explanation is that in this study, dependence was manipulated more successfully than was interference. Since there were no manipulation checks included in this study, this question remains unanswered, but the results of the pilot do not suggest that the dependence manipulation was more successful than the interference manipulation. In addition, in this study, separate $2 \times 2$ ANOVAs with interference and dependence as independent variables and two types of illness concerns as dependent variables show that although the interaction was not significant in any ANOVA, the means of these dependent variables were the highest in the high-dependence/high-interference condition, followed by either highdependence/low-interference or low-dependence/high-interference, and the lowest in the low-dependence/low-interference condition. 
Another explanation, however, is that being dependent on others as a consequence of a health problem can increase both individual and social illness concerns because being dependent on others could be interpreted as both losing independence (a personal concern) and being a burden on others (a social concern). This raises the question of whether dependence acted as a prime for both types of concerns being reported. This possibility suggests the importance of asking participants open-ended questions about the kinds of concerns they experience in the face of a health problem where no potential primes are present.

\section{Study 2}

Study 2 is designed to go beyond closed-ended questions of illness concerns to examine individuals' spontaneous descriptions of actual health problems in relation to different self-construals that are endorsed. In addition, in Study 2, illness-related concerns are examined in a real patient population with a wider age range, with the goal of having a more comprehensive understanding of the relation between self-construal and illness-related concerns applicable to the larger population.

To attain these goals, we asked a sample of individuals to tell us about a personal health problem in an open-ended format and respond to openended questions about the consequences that their health problem caused ${ }^{3}$ to examine whether their recollections varied as a function of the type of selfconstrual endorsed. Moreover, we also aim to check the validity of the closed-ended illness concern items developed in Study 1 by comparing them to individuals' open-ended accounts of their health problem. In this study, in which we asked volunteers to recall and share a past illness episode, our hypotheses were derived from the recent literature on self-construal and memory, which suggests that self-related information should be more detailed and better elaborated in the memories of people who possess an independent self-construal, whereas other-related information should be more detailed and better elaborated in the memories of people who have an interdependent self-construal (e.g., Markus \& Kitayama, 1991; Ng \& Zhu, 2001, Wang, 2001). Thus, we propose the following hypotheses:

Hypothesis 6. Independent self-construal will be associated with free recall of self-focused illness memories, whereas inter-

${ }^{3}$ In Study 2, we asked participants to report about the consequences of their health problem (instead of the concerns that they experienced during the course of the health problem) because we believed that the items developed for the Illness Concerns Scale in Study 1 tapped more into the potential consequences of the imagined health problem and that as a result of this conceptual match, it would make more sense to about consequences in Study 2 rather than concerns. 
dependent self-construal will be associated with free recall of other-focused illness memories.

Hypothesis 7. Independent self-construal will be associated with recall of personal illness-related consequences, whereas interdependent self-construal will be associated with recall of relational or social illness-related consequences.

In Study 2, we focused on independent and collective self-construals, following the results of Study 1, and did not measure relational selfconstrual. Also, we used a different self-construal scale in this study to examine if the results obtained in Study 1 would replicate with another measure.

\section{Method}

\section{Participants}

Participants were 73 adults (57 women, 16 men) who were recruited by means of the following strategies: recruitment on university campus (with posters or by advertising in adult student classes; $n=21$ ), advertisement in local newspapers $(n=25)$, and posters at various hospitals $(n=27)$. The mean age of the sample was 33.52 years $(S D=12.59)$ with a range of 17 to 72 years. The majority of the sample identified themselves as either Canadian $(n=32$; $43.84 \%)$ or European-Canadian $(n=26 ; 35.62 \%)$. The rest of the sample identified themselves as either East Asian-Canadian $(n=4 ; 5.5 \%)$, AfricanCanadian $(n=2 ; 2.74 \%)$, Middle Eastern-Canadian $(n=3 ; 4.11 \%)$, Jamaican-Canadian $(n=2 ; 2.74 \%)$, Indian-Canadian $(n=1 ; 1.37 \%)$, or of mixed ethnicity $(n=1 ; 1.37 \%)$. There were 2 participants who failed to indicate their ethnicity.

\section{Procedure}

The study was advertised as one focusing on how people remember past illness episodes. Volunteers who had a minor or major health problem that affected their life for at least 2 or 3 days at any point in the last 12 months were invited to participate. Volunteering participants were either handed or sent an envelope containing a consent form; a questionnaire; and a stamped, pre-addressed envelope to return the completed questionnaire. A second envelope was included in the package, in which participants were to insert and seal the signed consent form. 


\section{Study Materials}

Illness description task. In the illness description task, participants were asked to take a moment to think of an illness episode or injury that they had experienced within the last 12 months and that lasted for at least 2 to 3 days. Participants were asked to describe the episode in writing as precisely as they could. For this task, participants were given a blank sheet with instructions written across the top. At the bottom of the page, participants were asked to rate the seriousness of this health problem on a 7-point scale ranging from 1 (not serious at all) to 7 (extremely serious) and to indicate how long they had experienced this problem.

Open-ended questions about illness-related consequences. The two openended sections inquire about the consequences of the illness episode about which participants chose to write. One question is concerned with self-related consequences (i.e., "What consequences [short-term or long-term] did this event have for you?"), and the other is concerned with other-related consequences (i.e., "What consequences [short-term or long-term] did this event have for other people close to you?"). Participants had a half page to answer each question.

Closed-ended illness concerns scale. Participants were then given a modified version of the illness concerns scale that was used in Study 1. The illness concerns scale in Study 2 includes only those items that clearly loaded on either the social or the personal illness concern factor in Study 1. There were 27 items in total, 16 of which were social, and 11 of which were personal. ${ }^{4}$

Self-construal scales. After completing the open-ended sections, participants were given collective and independent self-construal scales. The first scale was the 10-item Collective-Interdependent Self-Construal Scale by Gabriel and Gardner (1999), which taps into individuals' relations with groups (e.g., "When I think of myself, I often think of groups that I belong to"). Singelis' (1994) 12-item Independent Self-Construal Scale ${ }^{5}$ measures the extent to which one's self is construed independently (e.g., "I act the same way no matter who I am with"). In both scales, respondents were asked to indicate their agreement with the items on a 7-point scale ranging from 1 (strongly disagree) to 7 (strongly agree). The reliability coefficient was .92

${ }^{4} \mathrm{An}$ item that successfully loaded on the social illness concerns factor was mistakenly left out in Study 1, which accounts for having 16 items instead of 17 in the social illness concern subscale in Study 2.

${ }^{5}$ Participants completed the independent and the interdependent subscales of Singelis' (1994) Self-Construal Scale. The interdependent subscale in this study had an unacceptable reliability coefficient and was found to have a multifactorial structure. Given that the scale was not measuring a clear construct that could be labeled interdependent self-construal, we are reporting the results that are related to the independent self-construal subscale only. 
$(M=4.13, S D=1.37)$ for the collective self-construal scale, and .68 $(M=5.09, S D=.77)$ for the independent self-construal scale.

\section{Coding of Open-Ended Responses}

The illness description task was content-analyzed for the frequency with which participants referred to themselves and to other people in their description of the health problem. To this end, a preliminary coding scheme was created with three main categories: illness-related statements, self-related statements, and other-related statements, which were refined as data coding took place. After initial coding, three further categories were added to capture meaningful differences between different kinds of self-related statements.

The first category included self-related statements referring to the psychological elements in the person's story, such as the person's emotions, thoughts, and dreams (Item 2a: psychological self-related statements). The second category included statements referring to other issues related to the person, such as the types of action the person took to deal with the problem or financial issues (Item 2b: other self-related statements). The third category included the statements that described the person in an interaction with another person or group (i.e., "I asked for help from my parents"; Item 2c: interpersonal statements; see Table 3). This last category included statements that were focused on the person himself or herself, despite the fact that they included a reference to a social other. Propositions coded under the otherrelated statements category, however, were exclusively about the effects of the illness on other individuals or groups.

These categories were then used to code every meaningful statement in participants' stories. A meaningful unit was defined as any proposition that gave distinct information about either the person, illness, or someone else. For example, the sentence "I have become dependent on medication and gained a lot of weight" was coded as conveying two distinct pieces of information (being dependent on medication and gaining a lot of weight). The categories were mutually exclusive such that each proposition was coded using only one category. Open-ended questions about illness-related consequences were coded using the same categories created for coding the illness description task (see Table 4).

Two coders, who were blind to study hypotheses, coded both illness descriptions and responses to open-ended questions. Disagreements in coding were resolved by discussing the inconsistencies between the two coders, and the coding scheme that was decided on with consensus was applied to relevant sections. Interrater reliability, as measured by Cohen's 
Table 3

Categories Used to Code the Self- and Other-Related Information in the Illness Description Task

\begin{tabular}{lcc}
\hline Category & Frequency & Percentage \\
\hline $\begin{array}{l}\text { 1. Illness body-related statements } \\
\text { Example: My sugar was over 210. }\end{array}$ & .339 & 26.4 \\
$\begin{array}{l}\text { 2a. Self-related statements (psychological) } \\
\text { Example: I was desperate. }\end{array}$ & 288 & 22.4 \\
$\begin{array}{l}\text { 2b. Self-related statements (other) } \\
\text { Example: My savings were all gone. }\end{array}$ & 491 & 38.2 \\
$\begin{array}{l}\text { 2c. Self-related statements (interpersonal) } \\
\begin{array}{l}\text { Example: I asked for help from my parents. } \\
\text { 3. Other-related statements }\end{array}\end{array}$ & 90 & 7.0 \\
$\begin{array}{l}\text { Example: My parents did not know what to do. } \\
\text { 4. Uncodable statements }\end{array}$ & 62 & 4.8 \\
\hline
\end{tabular}

Table 4

Categories Used to Code Consequences of the Health Problem

\begin{tabular}{lcc}
\hline Category & Frequency & Percentage \\
\hline $\begin{array}{l}\text { 1. Physical or illness-related consequences } \\
\text { Example: I have become dependent on }\end{array}$ & 87 & 35.0 \\
$\quad$ medication and gained a lot of weight. & & \\
2a. Self-related statements (psychological) & 51 & 20.6 \\
$\begin{array}{l}\text { Example: I suffered from anxiety for a long time. } \\
\text { 2b. Self-related statements (other) }\end{array}$ & 89 & 28.2 \\
$\begin{array}{l}\text { Example: Having to pay for costly drugs. } \\
\text { 3. Other-related consequences }\end{array}$ & 40 & 16.1 \\
Example: My son got affected very negatively. & & \\
\hline
\end{tabular}

kappa, which is a conservative estimate (e.g., Andren, 1981; Cohen, 1960), ranged between .61 and .79 in all open-ended sections. Percentage of agreement between coders ranged between $70 \%$ and $91 \%$ across different coding themes. 
Results

Duration and Seriousness of Health Problems

The duration of the health problems that participants wrote about ranged from 1 day to 20.3 years ( $M=14.7$ months, $S D=43.2$ months). The seriousness of the health problems was rated between 2 and $7(M=4.97, S D=1.42)$.

\section{Illness Description Task}

To examine whether type of self-construal endorsed was associated with type of information recalled (i.e., self- vs. other-related) as depicted in participants' illness stories (Hypothesis 6), a series of multiple regressions was conducted, with the relative number of recollections in each subcategory as criterion, and collective and independent self-construal as predictor variables. In all regressions, proportional frequencies (i.e., number of mentions of a specific category divided by total number of mentions) were used instead of the raw number of consequences mentioned in each subcategory. Results did not change when raw numbers were used instead.

All regression analyses were repeated controlling for duration, seriousness of the health problem, and age. None of the analyses reveal different results with these demographic variables added in the regression. The results show that only collective self-construal significantly predicted the proportion of other-related statements in participants' illness recollections $(\beta=.35$; $\left.R_{\text {adj. }}^{2}=.10\right), t(67)=3.08, p=.003, F(2,67)=4.93, p<.05$. Contrary to Hypothesis 6, none of the self-related categories in the illness description task were predicted by the endorsement of independent self-construal.

\section{Open-Ended Illness-Related Consequences}

Self-related consequences. To test the first part of Hypothesis 7-that independent self-construal would be associated with recall of self-related consequences - a multiple regression was conducted, with percentage of selfrelated consequences generated as the criterion, and collective and independent self-construal scales as predictors. This regression was not significant $\left(R_{\text {adj. }}^{2}=.01\right), F<1, n s$. None of the separate regressions with the proportional frequency of different categories of self-related consequences reveal a significant predictor, either (see Table 4). These results show that, contrary to our hypothesis, the independent self-construal scale did not contribute to the explanation of recall of self-related consequences. 
Other-related consequences. To test the second part of Hypothesis 7-that interdependent self-construal would be associated with recall of other-related consequences - a multiple regression was conducted with percentage of other-related consequences generated as the criterion, and collective and independent self-construal scales as predictors. The regression revealed an $R^{2}$ significantly different from $0\left(R_{\text {adj. }}^{2}=.10\right), F(2,70)=5.02$, $p=.009$, with collective self-construal being the only significant predictor $(\beta=.31), t(70)=2.77, p=.007$.

\section{Illness Concerns Scale}

The revised 27-item illness concerns scale was subjected to a principal axis factoring that suggested a three-factor solution in its scree plot. ${ }^{6}$ The factor analysis was repeated, forcing a three-factor solution and using an oblique rotation to allow the factors to correlate. Out of 17 interpersonal or grouprelated illness concern items, 15 loaded on the first factor, with factor loadings ranging from .40 to .74 . This factor was called the social illness concerns factor, as in Study 1. Out of 11 personal illness concern items, 7 loaded on the second factor, with factor loadings ranging from .54 to .91. This factor was labeled the personal illness concerns factor, as in Study 1. Two personal illness concern items loaded on the third factor, with loadings .86 and .68. These two items are "I was concerned this problem was getting in the way of my accomplishments" and "I was concerned this problem was getting in the way of fulfilling my potential." These items suggest that individuals' concerns about their achievement suffering as a consequence of the health problem they experienced emerged as a separate factor in this community sample. Therefore, this factor, called the achievement-related illness concerns factor, was examined in addition to the other two illness concern factors. This three-factor solution accounted for $57.2 \%$ of the total variance, with the first factor accounting for $38.4 \%$ of the total variance, the second factor accounting for an additional $11.8 \%$, and the third factor contributing $7.0 \%$ to the explanation of total variance.

Three illness concern scales were computed with the items that clearly loaded on each factor. The first illness concern scale (social illness concerns scale) was composed of 15 interdependent illness concern items and had a reliability of $.91(M=3.65, S D=1.38)$. The second illness concern scale (personal illness concerns scale) consisted of 7 independent illness concern items with a reliability coefficient of $.91(M=4.61, S D=1.62)$. Finally, the

${ }^{6}$ The factor structure of the illness concerns scale in Study 2 should be read with caution because of the small sample size. 
Table 5

Correlations Between Variables: Study 2

\begin{tabular}{lllll}
\hline Variable & 1 & 2 & 3 & 4 \\
\hline 1. Collective Self-Construal Scale & - & & & \\
2. Independent Self-Construal Scale & .09 & - & & \\
3. Social illness concerns & $.24^{*}$ & -.14 & - & \\
4. Personal illness concerns & .13 & -.10 & $.53^{* *}$ & - \\
5. Achievement-related illness concerns & .19 & -.14 & $.39^{* *}$ & .17 \\
\hline$*_{p<.05 . * *}^{*} p<.01$. & & & &
\end{tabular}

third scale (achievement-related illness concerns scale) consisted of the two achievement-related concern items and had a reliability of $.82(M=5.32$, $S D=1.78$; see Table 5 for correlations among all study variables). The achievement-related concerns negatively correlated with age $(r=-.39$, $p=.001)$, and students $(M=5.66)$ scored higher on this scale than did nonstudents $(M=4.75), F(1,70)=4.62, p=.035$.

We examined the relationship between these three illness concern subscales and the self-construal scales included in this study to see if the relationships reported in Study 1 would be replicated in this sample. Three separate multiple regression analyses were run with the three illness concern scale scores as the criteria, and collective and independent self-construal scores as predictors. Collective self-construal significantly predicted scores on the social illness concerns scale $\left(\beta=.26 ; R_{\text {adj. }}^{2}=.08\right), t(70)=2.22, p=.03, F(2$, $70)=3.17, p<.05$. The other two illness concern subscales were not predicted by any of the self-construal scales.

\section{Open-Ended and Closed-Ended Questions of Illness Concerns}

One of the reasons why the closed-ended illness concerns scale was included in this study was to examine whether participants' open-ended accounts of their illness concerns were in line with their responses to the closed-ended questions of illness consequences. If this were true, it would be expected that participants' personal illness concern scores, as measured by the closed-ended scale, would be positively associated with the recall of health-problem-related consequences that are individualistic in nature. Similarly, one's score on the social illness concerns scale would be expected to be 
positively associated with the recall of health-problem-related consequences that concerned others, rather than the self.

To examine these hypotheses, zero-order correlations were run with the percentage of self-related and other-related consequences generated by participants and the two illness-related concern scales. The social illness concerns scale was positively and significantly correlated with the number of other-related consequences generated $(r=.37, p=.001)$. The personal illness concerns scale was not associated with the number of self-related consequences generated.

As outlined earlier, self-related consequences included self-statements of different nature (see Table 3). Two self-related categories-namely, interpersonal self-related consequences and psychological self-related consequences - were examined separately for their relation to the two illness concern scales, because they were deemed more likely to reflect the characteristics of one's self-construal than were other self-related categories that contained information about physical or financial-/work-/school-related consequences. Because these subcategories were highly skewed, however, they were turned into categorical variables with two levels; one representing those who generated a concern in that subcategory, and the other representing those who did not. It was expected that those who generated psychological self-related consequences would score higher on the personal illness concerns scale than those who did not mention any of these concerns, and those who generated interpersonal self-related consequences would score higher on the social illness concerns scale.

Two separate ANOVAs were conducted to examine these hypotheses. The first ANOVA, with the personal illness concerns scale as the dependent variable and the categorical psychological self-related consequences as the independent variable, reveals an effect that was significant at the trend level, $F(1,71)=2.86, p=.095$, such that those who generated psychological selfrelated consequences tended to score higher $(M=4.96)$ on the personal illness concern scale than those who did not $(M=4.32)$. The second ANOVA, with the social illness concerns scale as the dependent variable and the categorical interpersonal self-related consequences as the independent variable, reveals a significant effect, $F(1,71)=6.74, p=.011$, such that those who generated interpersonal self-related consequences scored significantly higher $(M=4.19)$ on the social illness concern scale than those who did not $(M=3.35)$.

\section{Discussion}

Study 2 was designed to uncover the relationship between people's selfconstrual and both the nature of their general recollections of a past health 
problem and their specific recollections of illness-related consequences experienced during the course of that health problem. Another goal was to examine whether the results of Study 1 would replicate in a sample of people with real past health problems who responded to similar questions in an open-ended format.

\section{Self-Construal and Illness Recollections}

First, it is worth mentioning that most recollections referred to illness or body-related issues. This is an expected outcome since our recruitment procedure might have operated as a prime to make people focus more on physical characteristics of their health problem. Issues related to the self followed in frequency and were in turn followed by statements related to issues about others. This ordering suggests that memories related to physical health or to a specific health problem were both more detailed and more easily retrieved from memory than were issues related to the self or social others.

The analyses examining the relationship between type of self-construal endorsed and type of recollections (i.e., self- or other-related) people had when asked to recall a past health problem show that independent selfconstrual was not related to any type of recollections. However, collective self-construal predicted the extent to which people mentioned issues related to others in their free-recall illness descriptions and the number of otherrelated consequences that study participants generated when they were specifically asked about them. In other words, collective self-construal predicted the reporting of social illness concerns in a task that required spontaneous recollection aspects of the health problem and that is, therefore, considered a more sensitive test of what is more easily accessible in people's memory and how elaborate information is stored in memory, as well as in a more structured task in which people may respond by trying to retrieve information consistent with what is asked.

The relationship found between the illness concern scales and different types of self-construal in Study 1 was replicated only to a certain extent in Study 2. In Study 1, independent self-construal was found to marginally predict the experience of personal illness concerns, as measured by the closedended scale; and collective self-construal was found to predict social illness concerns, as measured by the closed-ended scale. In Study 2, only collective self-construal was found to predict other-related recollections from a past health problem. This overlap between Studies 1 and 2 was observed, despite the use of a different collective self-construal scale in the two studies.

A potential explanation for the lack of prediction by independent selfconstrual in the criterion variables discussed previously is that in a real illness 
context-compared to an imagined illness context-self-related consequences are experienced by everyone, regardless of how strongly they endorse independent self-construal. In the end, a physical health problem is first about the person who experiences the immediate physical and psychological consequences associated with the health problem. Other life events, such as childhood memories (e.g., Wang, 2001), might provide a more sensitive context for a distinction between the type of recollections of those who endorse a strong independent self-construal and those who endorse a strong interdependent self-construal.

\section{Factor Structure of Illness Concerns}

The samples and study designs of Studies 1 and 2 differed in many ways. Study 1's sample consisted of university students who were asked to imagine themselves having a health problem. Study 2's sample consisted mostly of participants from the community who were asked to recall a real health problem that they had experienced in the past. Nevertheless, despite these differences and the small sample size in Study 2, the factor structure of illness concern items obtained in Studies 1 and 2 was remarkably similar.

Most items that clearly fell under either the social illness concern factor or the personal illness concern factor in Study 1 also loaded on the same factors in Study 2. One major difference is that in Study 2, a third factor emerged, consisting of two items tapping into concerns related to achievement. This may have been related to the characteristics of the samples in Studies 1 and 2. The fact that age negatively correlated with achievement-related concerns and that students scored higher on this concern than non-students points to this possibility. Since the sample in Study 1 was less diverse in terms of age and profession (i.e., the majority of the sample consisted of undergraduate students), this may have not emerged as a separate factor. Another potential explanation is a methodological one; namely, that this factor may have been obscured by the presence of additional concern items in Study 1 that were omitted in Study 2.

\section{Open-Ended and Closed-Ended Illness Concerns}

The present study provided the means to assess the similarity between individuals' responses to open-ended and closed-ended questions concerning their concerns about the health problem. The results provide external validation to the illness concerns scale, in that participants recalled types of information from their past health problem that were consistent with their 
responses to the closed-ended questions on illness-related consequences. Scores on social illness concerns and the number of other-related consequences generated were positively correlated. Further, the findings show that participants who generated psychological self-related consequences tended to score higher on the personal illness concern scale than those who did not, and participants who generated interpersonal self-related consequences scored higher on the social illness concern scale than those who did not.

In a final analysis, Study 2 provides evidence for the link between selfconstrual and people's general and specific recollections concerning a health problem experienced in the past. Moreover, the findings also contribute to the scarce literature on the link between self-construal and memory by providing evidence from a domain-specific aspect of autobiographical memory, and add to previous evidence indicating that one's self-construal shapes the way autobiographical information is processed, organized, retained, and retrieved (e.g., Wang, 2001, 2004). We believe that testing the relationship between self-construal and memory is likely to be more stringent when people are asked to remember a past health problem, as the focus of attention is likely to be on one's body and health. The fact that significant relationships were observed in the way people's self was construed and the type of information they remembered from a past health problem suggests that even in highly person-bound contexts, one's self-construal may influence what one recalls from the past.

\section{General Discussion}

Although social psychological concepts have been examined in healthrelated research for some time, relatively little attention has been paid to the role that the self plays in health and disease, or in moderating individuals' health-related behaviors (Kihlstrom \& Kihlstrom, 1999). For their part, Ashmore and Contrada (1999) argued that there is an apparent disconnection between models of self regulation in health psychology and the treatment of self by psychologists interested in self and social identity. The two studies reported here examined a specific aspect of the self-concept; namely, the extent to which others are incorporated into the self, in relation to the nature of concerns individuals may experience in the face of imagined and real physical health problems.

Study 1 showed that interdependent self-construal was associated with the experience of social illness concerns, whereas independent self-construal tended to be associated with the experience of personal illness concerns in an imagined illness context. Study 2 showed that interdependent self-construal was associated with the recall of other-related information from a past health 
problem, as well as the recall of other-related consequences. In addition to having contributed to research on self and physical health, we believe that the present two studies can also inform us regarding potential applied consequences of endorsing different self-construals in health settings.

\section{Implications for Applied Settings}

The findings of Study 1 show that the endorsement of interdependent self-construal predicts the experience of social illness concerns. This suggests that assuming that individuals with health problems are only concerned with their personal physical and psychological well-being may not represent the entire picture of what worries an individual in the face of a health problem. As previous research has shown, potential issues that concern other individuals in one's life can be a determining factor in patients' choices about the timing and type of action taken to deal with a health problem (e.g., Uskul, Ahmad, Leyland, \& Stewart, 2003).

Acknowledging and addressing the different kinds of concerns individuals may experience would be expected to be of vital use in communicating effectively with a patient. Addressing such issues in the course of a health problem could increase the effectiveness of physician-patient communication or communication employed in health campaigns and in educational materials, which in turn may help to encourage taking preventive action and seeking medical help in a timely fashion, and increase adherence to treatment. Research concerning the effects of health communication that focuses solely on the physical and individual consequences of engaging in a risky health behavior or taking preventive action and medical adherence versus health communication that incorporates both the individual and his or her social environment is needed in order to translate the findings presented in the current studies into applied issues, such as design of health campaigns and physician-patient communication (Uskul \& Oyserman, 2006). In addition, examining how health communication with different foci is perceived and acted upon among individuals who endorse different kinds of selfconstrual is believed to be useful for designing tailored and more effective messages. Communicating with individuals while placing them in a social context that they care about might be especially important for those who endorse a strong interdependent self-construal. Finally, examining whether different types of illness-related concerns differ in terms of the behavioral consequences they cause would be worthwhile. Individuals might choose to take immediate action or to delay seeking medical help, depending on the nature of the concerns the health problem evokes in their minds. 


\section{Future Directions}

A natural extension of these studies is to examine whether there are cross-cultural differences in the health-related phenomena investigated here and, if so, whether self-construal would be a successful mediator between culture and these phenomena. Most certainly, when cultures are compared in terms of health and illness-related issues, structural characteristics of the societies - such as their health and social support systems - would need to be taken into account, as these can definitely affect the nature of concerns individuals might have when they face a health problem.

Another interesting direction to follow is the question of how people with different self-concepts come to differ in the degree to which they feel concerned about others when facing health problems and remember otherrelated information from past illnesses. Past research has shown that children in collectivistic societies are socialized to maintain social harmony, be empathetic, and avoid bothering others (e.g., Lebra, 1994; Rothbaum, Pott, Azuma, Miyake, \& Weisz, 2000; White \& LeVine, 1986). Whether this socialization is extended to children's perceptions and experience of health-related issues requires further research. A way in which this question could be explored is by examining issues such as how parents communicate with their children about health and safety, whether parents with backgrounds that emphasize independence and interdependence to different extents have the same beliefs about why their children should be healthy and safe, and whom parents believe is responsible for their child's health.

\section{References}

Aiken, L. S., \& West, S. G. (1991). Multiple regression: Testing and interpreting interactions. Thousand Oaks, CA: Sage.

Andren, G. (1981). Reliability and content analysis. In K. E. Rosengren (Ed.), Advances in content analysis (pp. 43-69). London: Sage.

Aron, A., Aron, E. N., \& Smollan, D. (1992). Inclusion of other in the self scale and the structure of interpersonal closeness. Journal of Personality and Social Psychology, 63, 596-612.

Aron, A., \& McLaughlin-Volpe, T. (2001). Including others in the self: Extensions to own and partner's group membership. In C. B. Sedikides \& M. B. Brewer (Eds.), Individual self, relational self, collective self (pp. 89-108). Philadelphia: Psychology Press.

Ashmore, R. D., \& Contrada, R. J. (1999). Conclusion: Self, social identity, and the analysis of social and behavioral aspects of physical health and illness. In R. J. Contrada \& R. D. Ashmore (Eds.), Self, social identity, 
and physical health: Interdisciplinary explorations (pp. 240-255). New York: Oxford University Press.

Bakan, D. (1966). The duality of human existence. Boston: Beacon Press.

Baumeister, R. F., \& Leary, M. R. (1995). The need to belong: Desire for interpersonal attachments as a fundamental human motivation. Psychological Bulletin, 117, 497-529.

Bishop, G. D. (1987). Lay conceptions of physical symptoms. Journal of Applied Social Psychology, 17, 127-146.

Boehnke, K., Schwartz, S., Stromberg, C., \& Sagiv, L. (1998). The structure and dynamics of worry: Theory, measurement, and cross-national replications. Journal of Personality, 66, 745-782.

Boehnke, K., Stromberg, C., Regmi, M. P., Richmond, B. O., \& Chandra, S. (1998). Reflecting the world "out there": A cross-cultural perspective on worries, values, and well-being. Journal of Social and Clinical Psychology, $17,227-247$.

Brewer, M. B., \& Gardner, W. (1996). Who is this "we"? Levels of collective identity and self-representations. Journal of Personality and Social Psychology, 71, 83-93.

Brockner, J., \& Chen, Y. R. (1996). The moderating roles of self-esteem and self-construal in reaction to a threat to the self: Evidence from the People's Republic of China and the United States. Journal of Personality and Social Psychology, 71, 603-615.

Cohen, J. (1960). A coefficient of agreement for nominal scales. Educational and Psychological Measurement, 20, 37-46.

Cross, S. E., \& Madson, L. (1997). Models of the self: Self-construals and gender. Psychological Bulletin, 122, 5-37.

Davey, G. C. L., \& Tallis, F. (1994). Worrying: Perspectives on theory, assessment, and treatment. New York: John Wiley \& Sons.

Frijda, N. H. (1986). The emotions. New York: Cambridge University Press.

Fritz, H. L. (2000).Gender-linked personality traits predict mental health and functional status following a first coronary event. Health Psychology, 19, 420-428.

Gabriel, S., \& Gardner, W. (1999). Are there "his" and "hers" types of interdependence? The implications of gender differences in collective versus relational interdependence for affect, behavior, and cognition. Journal of Personality and Social Psychology, 77, 642-655.

Geertz, C. (1975). On the nature of anthropological understanding. American Scientist, 63, 47-53.

Gilligan, C. (1982). In a different voice: Psychological theory and women's development. Cambridge, MA: Harvard University Press.

Gudykunst, W. B., Matsumoto, Y., Ting-Toomey, S., Nishida, T., Kim, K., \& Heyman, S., et al. (1996). The influence of cultural individualism- 
collectivism, self-construals, and individual values on communication styles across cultures. Human Communication Research, 22, 510-543.

Helgeson, V. S. (1990). The role of masculinity as a prognostic predictor of heart attack severity. Sex Roles, 22, 755-774.

Helgeson, V. S. (1995). Masculinity, men's roles, and coronary heart disease. In D. F. Sabo \& D. F. Gordon (Eds.), Men's health and illness: Gender, power, and the body (pp. 68-104). Thousand Oaks, CA: Sage.

Helgeson, V. S., \& Fritz, H. L. (1996). Implications of unmitigated communion and communion for adolescent adjustment to Type I diabetes. Women's Health: Research on Gender, Behavior, and Policy, 2, 163188.

Hui, C. H. (1988). Measurement of individualism-collectivism. Journal of Research in Personality, 22, 17-36.

Jiménez, M., \& Lunman, K. (2004, August 19). Canada's biggest cities see influx of new immigrants. The Globe and Mail, p. A1.

Kanagawa, C., Cross, S. E., \& Markus, H. R. (2001). "Who am I?" The cultural psychology of the conceptual self. Personality and Social Psychology Bulletin, 27, 90-103.

Kashima, E. S., \& Hardie, E. A. (2000). The development and validation of the Relational, Individual, and Collective Self-Aspects (RIC) Scale. Asian Journal of Social Psychology, 3, 19-48.

Kashima, Y., Kashima, E., \& Aldridge, J. (2001). Toward cultural dynamics of self-conceptions. In C. Sedikides \& M. B. Brewer (Eds.), Individual self, relational self, collective self (pp. 277-298). Philadelphia: Psychology Press.

Kashima, Y., Yamaguchi, S., Kim, U., Choi, S. C., Gelfand, J. M., \& Yuki, M. (1995). Culture, gender, and self: A perspective from individualismcollectivism research. Journal of Personality and Social Psychology, 69, 925-937.

Kihlstrom, J., \& Kihlstrom, L. C. (1999). Self, sickness, somatization, and systems of care. In R. J. Contrada \& R. D. Ashmore (Eds.), Self, social identity, and physical health: Interdisciplinary explorations (pp. 23-42). London: Oxford University Press.

Lazarus, R. S. (1991). Emotion and adaptation. New York: Oxford University Press.

Lebra, T. S. (1994). Mother and child in Japanese socialization: A JapanU.S. comparison. In P. M. Greenfield \& R. R. Cocking (Eds.), Crosscultural roots of minority child development (pp. 259-274). Hillsdale, NJ: Lawrence Erlbaum.

Leventhal, H., Diefenbach, M., \& Leventhal, E. A. (1992). Illness cognition: Using common sense to understand treatment adherence and affect cognition interactions. Cognitive Therapy and Research, 16, 143-163. 
Leventhal, H., Nerenz, D. R., \& Steele, D. J. (1984). Illness representations and coping with health threats. In A. Baum, S. E. Taylor, \& J. E. Singer (Eds.), Handbook of psychology and health (pp. 219-252). Hillsdale, NJ: Lawrence Erlbaum.

Lipowski, Z. J. (1985). "What does the word 'psychosomatic' really mean? A historical and semantic inquiry": Reply. Psychosomatic Medicine, 47, 67-69.

Loevinger, J. (1976). Origins of conscience. Psychological Issues, 9, 265-297. Lucock, M. P., \& Morley, S. (1996). The Health Anxiety Questionnaire. British Journal of Health Psychology, 1, 137-150.

Markus, H. R., \& Kitayama, S. (1991). Culture and the self: Implications for cognition, emotion, and motivation. Psychological Review, 98, 224-253.

Matsumoto, D., Weissman, M. D., Preston, K., Brown, B. R., \& Kupperbusch, C. (1997). Context-specific measurement of individualismcollectivism on the individual level: The Individualism-Collectivism Interpersonal Assessment Inventory. Journal of Cross-Cultural Psychology, 28, 743-767.

McAdams, D. P. (1995). What do we know when we know a person? Journal of Personality, 63, 365-396.

Mesquita, B. (2001). Emotions in collectivist and individualist contexts. Journal of Personality and Social Psychology, 80, 68-74.

Meyer, D., Leventhal, H., \& Gutmann, M. (1985). Common-sense models of illness: The example of hypertension. Health Psychology, 4, 115-135.

Ng, S. H., \& Zhu, Y. (2001). Attributing causality and remembering events in individual- and group-acting situations: A Beijing, Hong Kong, and Wellington comparison. Asian Journal of Social Psychology, 4, 39-52.

Oyserman, D., Coon, H., \& Kemmelmeier, M. (2002). Rethinking individualism and collectivism: Evaluation of theoretical assumptions and metaanalyses. Psychological Bulletin, 128, 3-72.

Oyserman, D., \& Lee, S.W. (in press). Priming "culture": Culture as situated cognition. In S. Kitayama \& D. Cohen (Eds.), Handbook of cultural psychology. New York: Guilford.

Rothbaum, F., Pott, M., Azuma, H., Miyake, K., \& Weisz, J. (2000). The development of close relationships in Japan and the United States: Paths of symbiotic harmony and generative tension. Child Development, 71, $1121-1142$.

Shweder, R. A., \& Bourne, E. J. (1982). Does the concept of the person vary cross-culturally? In J. Marsalla \& G. White (Eds.), Cultural concepts of mental health and therapy (pp. 97-137). Boston: Reidel.

Singelis, T. M. (1994). The measurement of independent and interdependent self-construals. Personality and Social Psychology Bulletin, 20, 580591. 
Singelis, T., Triandis, H., Bhawuk, D., \& Gelfand, M. (1995). Horizontal and vertical dimensions of individualism and collectivism: A theoretical and measurement refinement. Cross-Cultural Research: The Journal of Comparative Social Science, 29, 240-275.

Smith, T. (1992). Hostility and health: Current status of a psychosomatic hypothesis. Health Psychology, 11, 139-150.

Tajfel, H., \& Turner, J. C. (1979). An integrative theory of intergroup conflict. In W. G. A. S. Worchel (Ed.), The social psychology of intergroup relations (pp. 33-47). Monterey, CA: Brooks-Cole.

Triandis, H. (1989). The self and social behavior in differing cultural contexts. Psychological Review, 96, 506-520.

Triandis, H. C. (1995). Individualism and collectivism. Boulder, CO: Westview.

Triandis, H., Bontempo, R., Betancourt, H., Bond, M., Leung, K., \& Brenes, A., et al. (1986). The measurement of the etic aspects of individualism and collectivism across cultures. Australian Journal of Psychology, 38, 257267.

Triandis, H. C., \& Gelfand, M. J. (1998). Converging measurement of horizontal and vertical individualism and collectivism. Journal of Personality and Social Psychology, 74, 118-128.

Triandis, H. C., McCusker, C., \& Hui, C. H. (1990). Multimethod probes of individualism and collectivism. Journal of Personality and Social Psychology, 59, 1006-1020.

Uskul, A. K., Ahmad, F., Leyland, N., \& Stewart, D. (2003). Hysterectomy and women's decision making. Women and Health, 38, 53-67.

Uskul, A. K., Hynie, M., \& Lalonde, R. N. (2004). Interdependence as a mediator between culture and interpersonal closeness for EuroCanadians and Turks. Journal of Cross-Cultural Psychology, 35, 174 191.

Uskul, A. K., \& Oyserman, D. (2006). Are "I" and "We" convinced by different health messages? Manuscript submitted for publication.

Wang, Q. (2001). Culture effects on adults' earliest childhood recollection and self-description: Implications for the relation between memory and the self. Journal of Personality and Social Psychology, 81, 220-233.

Wang, Q. (2004). The emergence of cultural self-constructs: Autobiographical memory and self-description in European American and Chinese children. Developmental Psychology, 40, 3-15.

White, M. I., \& LeVine, R. A. (1986). What is an ii ko (good child)? In H. Stevenson, H. Azuma, \& K. Hakuta (Eds.), Child development and education in Japan (pp. 55-62). New York: Freeman.

Wiggins, J. S. (1991). Agency and communion as conceptual coordinates for the understanding and measurement of interpersonal behavior. In 
W. M. Grove \& D. Cicchetti (Eds.), Thinking clearly about psychology (pp. 89-113). Minneapolis, MN: University of Minnesota Press.

Youniss, J., \& Smollar, J. (1985). Adolescent relations with mothers, fathers, and friends. Chicago: University of Chicago Press.

\section{Appendix}

\section{Illness Concerns Scale}

Personal concern items:

1. Losing the ability to do things for myself

2. Not being able to do things on my own

3. Not being able to rely on myself

4. Not being able to take care of myself

5. Having to depend on others for their help

6. Needing to ask for help from others

7. This problem getting in the way of fulfilling my potential (A)

8. Not being able to be my own boss

9. Not being able to fulfill my personal responsibilities*

10. This problem getting in the way of my accomplishments (A)

11. Losing my ability to do things that make me unique

Social concern items:

1. Not living up to expectations of the social group(s) I belong to (C)

2. Not being useful in the social group(s) I belong to (C)

3. Not being able to fulfill my responsibilities I have towards my social group(s) (C)

4. Being a burden on people with whom I share the same social group (s) (C)*

5. Not being able to be caring and attentive to people in my social group(s) (C)

6. Losing the ability to do things for people in my social group(s) (C)

7. Being a source of unhappiness for the members of my social group(s) (C)

8. The well-being of those with whom I share the same social group(s) (C)

9. Losing the good relationships I have with people in my social group(s) (C)

10. Experiencing a decrease of my status in the social groups I belong to $(\mathrm{C})$

11. Experiencing a decrease in my status among people close to me (R) 
12. Not living up to expectations of people who are close to me (R)

13. Losing the good relationships I have with people close to me (R)

14. Not being able to fulfill my close relationship responsibilities (R)

15. Not being useful in my close relationships $(\mathrm{R})$

16. Not being able to be caring and attentive to people who are close to me $(\mathrm{R})^{*}$

17. Being a source of unhappiness for those who are close to me (R)

Note. In Study 1, the items were presented after participants read the hypothetical illness scenario. The items followed the opening statement "In such a situation, I would be concerned about..." In Study 2, participants were asked to respond to the items by thinking of the illness episode they had just written about. The following instruction preceded the items: "In the illness situation I described above, I was concerned about..." In both studies, participants used a 7-point Likert scale ranging from 1 (not at all) to 7 (extremely).

*Items that did not load successfully on the expected factors in Study 2. (A) = Items loaded on a third factor in Study 2 that we called the achievement factor $;(\mathrm{C})=$ collective $($ group-related) concerns; $(\mathrm{R})=$ relational (interpersonal) concerns. 